\title{
Quench Protection Study of the Updated MQXF for the LHC Luminosity Upgrade (HiLumi LHC).
}

\author{
Vittorio Marinozzi, Giorgio Ambrosio, Paolo Ferracin, Susana Izquierdo Bermudez, Juho Rysti, \\ Tiina Salmi, Massimo Sorbi and Ezio Todesco
}

\begin{abstract}
In 2023, the LHC luminosity will be increased, aiming at reaching $3000 \mathrm{fb}-1$ integrated over 10 years. In order to obtain this target, new Nb3Sn low- $\beta$ quadrupoles (MQXF) have been designed for the interaction regions. These magnets present a very large aperture $(150 \mathrm{~mm}$, to be compared with the $70 \mathrm{~mm}$ of the present NbTi quadrupoles), and a very large stored energy density $\left(120 \mathrm{MJ} / \mathrm{m}^{3}\right)$. For these reasons, quench protection is one of the most challenging aspects of the design of these magnets. In fact, protection studies of a previous design showed that the simulated hot spot temperature was very close to the maximum allowed limit of $350 \mathrm{~K}$; this challenge motivated improvements in the current discharge modeling, taking into account the so-called dynamic effects on the apparent magnet inductance. Moreover, quench heaters design has been studied going into more details. In this paper, a protection study of the updated MQXF is presented, benefitting from the experience gained by studying the previous design. A study of the voltages between turns in the magnet is also presented during both normal operation and most important failure scenarios.
\end{abstract}

Index Terms - Niobium compounds, quench protection, superconducting accelerators.

\section{INTRODUCTION}

$\mathrm{T}$ THE Large Hadron Collider (LHC) in 2010-2013 has produced collisions between proton beams with up to 4 $\mathrm{TeV}$ energy per beam. Now, LHC is producing collisions at $6.5 \mathrm{TeV}$ per beam, and it will run until 2023. It is expected that in 2023 the integrated luminosity of 300 $\mathrm{fb}^{-1}$ will be reached; beyond this value, running the machine will not lead to significant statistical advantage, maintaining the present peak luminosity of $1.5-2 \times 10^{34}$ $\mathrm{cm}^{-2} \mathrm{~s}^{-1}$. Then, a luminosity upgrade program, named High Luminosity LHC (HL-LHC), has been planned, aiming at reaching $3000 \mathrm{fb}^{-1}$ in 10 years after 2025 . The main action will be reducing the beam size, and this can be achieved with more performing magnets in the interaction regions. In particular, the present low- $\beta$ triplet $\mathrm{NbTi}$ superconducting quadrupoles are planned to be substituted with new $\mathrm{Nb}_{3} \mathrm{Sn}$ magnets, called MQXF. It is one of the first $\mathrm{Nb}_{3} \mathrm{Sn}$ superconducting magnets designed in order to be inserted

V. Marinozzi and M. Sorbi are with University of Milano and INFNLASA, Milan, Italy, email: vittorio.marinozzi@mi,infn,it.

G. Ambrosio is with Fermi National Accelerator Laboratory, Batavia, IL 60510-5011, USA, email: giorgioa@ fnal.gov.

T. Salmi is with Tampere University of Technology, Tampere, Finland, email: tiina.salmi@tut.fi.

S. I. Bermudez, P. Ferracin, J. Rysti and E. Todesco are with CERN, TE-Dep, Geneve, Switzerland, email: ezio.todesco@cern.ch.

The research leading to these results has received funding from the European Commission under the FP7 project HiLumi LHC, GA no. 284404, co-funded by the DoE, USA and KEK, Japan. in a particle accelerator, and many challenges have been faced.

The quench protection of MQXF has been one of the most challenging aspects in the design, because of the large magnetic energy stored into the coils $\left(\sim 0.12 \mathrm{~J} / \mathrm{mm}^{3}\right.$, a factor 2 larger than in the present NbTi LHC low- $\beta$ quadrupoles), and of the high magnetic field $(\sim 12 \mathrm{~T})$ needed for the beams focusing. In fact, the first quench protection studies showed that the magnet safety could not be ensured, because the hot spot temperature was very close, or even larger than the maximum allowed of $350 \mathrm{~K}$ [1]. For this reason, new protection studies have been carried on, improving the quench heaters design, and studying with more detail the behaviour of the current decay, adding the simulation of dynamic effects on the magnet inductance, due to the coupling currents between the filaments. This study led to better results, showing that the magnet protection was possible [2]

Nevertheless, in the last year, the main magnet operation parameters have been upgraded, for example reducing the current and the cable dimensions, and some uncertain parameters have been established. The upgraded parameters are reported in Table I.

TABLE I MAIN PARAMETERS OF MQXF

\begin{tabular}{lc}
\hline \hline Aperture diameter & $150 \mathrm{~mm}$ \\
Gradient & $132.6 \mathrm{~T} / \mathrm{m}$ \\
Nominal current & $16470 \mathrm{~A}$ \\
Magnetic stored energy & $1.17 \mathrm{MJ} / \mathrm{m}$ \\
Inductance & $8.3 \mathrm{mH} / \mathrm{m}$ \\
Magnetic length (Q1/Q3) & $2 \times 4.2 \mathrm{~m}$ \\
Magnetic length (Q2a/Q2b) & $7.15 \mathrm{~m}$ \\
Conductor peak field & $11.4 \mathrm{~T}$ \\
Operating temperature & $1.9 \mathrm{~K}$ \\
Strand diameter & $0.850 \mathrm{~mm}$ \\
Bare cable width & $17.86 \mathrm{~mm}$ \\
Bare cable thin/thick edge thickness & $1.462 / 1.588 \mathrm{~mm}$ \\
Insulation thickness & $0.145 \mathrm{~mm}$ \\
Number of strands & 40 \\
Cu/Sc & 1.2 \\
Copper RRR & 100 \\
\hline \hline
\end{tabular}

Moreover, the option of protecting the whole triplet with a single protection system is under discussion. The triplet is constituted by four cold masses (Q1, Q2a, Q2b and Q3), and until now one protection circuit for Q1/Q3 and another one for $\mathrm{Q} 2 \mathrm{a} / \mathrm{Q} 2 \mathrm{~b}$ were foreseen. The option of Q1, Q2a, $\mathrm{Q} 2 \mathrm{~b}$ and Q3 in series could reduce costs, because only one power supply and just one dump resistor could be needed instead of two.

In this paper, the protection study of the upgraded version of MQXF is discussed. The analysis of the hot spot temperature is showed together with the analysis of the peak voltages during a quench. Nominal cases are analysed and compared to some quench heaters failure scenarios. 
Also, the option of protecting the triplet with a single protection circuit is compared to the nominal case of two circuits. The option of protecting MQXF using CLIQ [3] is not discussed in this paper; nevertheless, it is a solution under discussion [4]. CLIQ is a novel passive method for the quench protection, which take advantage of coupling currents in order to induce the quench in the whole magnet.

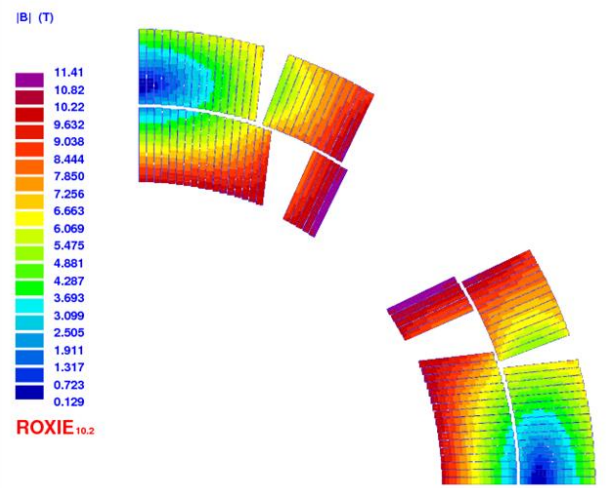

Fig. 1. One quadrant cross section of MQXF with magnetic field map.

\section{QUENCH HEATERS DESIGN}

MQXF stores very high magnetic energy at operation current (see Table 1). Moreover, it is a long magnet (magnetic length of Q1 and Q3 is 2x4.2 m, Q2a and Q2b $7.15 \mathrm{~m}$ ), therefore it has a large inductance. For these reasons, it is impossible to protect it using just a dump resistor, which is limited by the maximum voltage across the coil ends, and efficient quench heaters are needed. Previous studies [1] showed that heaters only on the outer layer are insufficient to assure magnet safety, therefore various designs for the inner layer heaters have been proposed. The heater designs used for the protection analysis showed in this paper are described below.

\section{A. Outer layer quench heaters}

The outer layer protection heaters are constituted of a set of simple straight strips that span along the coil and across each (pole and mid-plane) winding block. There are stainless steel heating stations, which have a width of 40 $\mathrm{mm}$ and are separated by $120 \mathrm{~mm}$ long copper-plated bridges. The design layout is showed in Fig. 2.

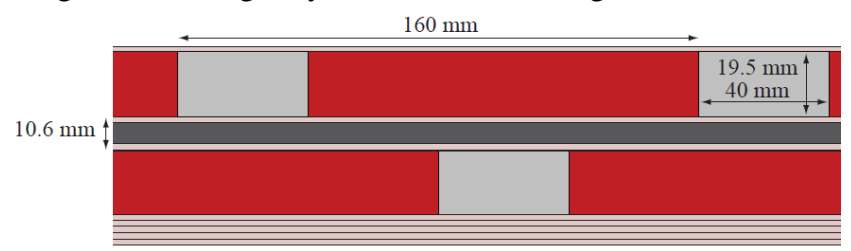

Fig. 2. Design of the MQXF outer layer protection heaters.

\section{B. Inner layer quench heaters}

The inner layer protection heaters are constituted of copper-plated narrow bridges, which connect wide stainless steel heating stations. This design is optimized in order to avoid as much as possible the helium bubbles issue [5]: the magnet inner layer is in direct contact with super-fluid helium, which could penetrate between the heaters strips and the coils; during a quench, the helium evaporates, and it generates bubbles, which reduces the thermal contact between the heater and the coil surface, reducing the heater efficiency. The design with narrow bridges should allow for more spacing for perforations, which should help the helium gas to evacuate the coil. The "snake" shape allows covering both the mid-plane and the pole blocks. This design is showed in Fig. 3.

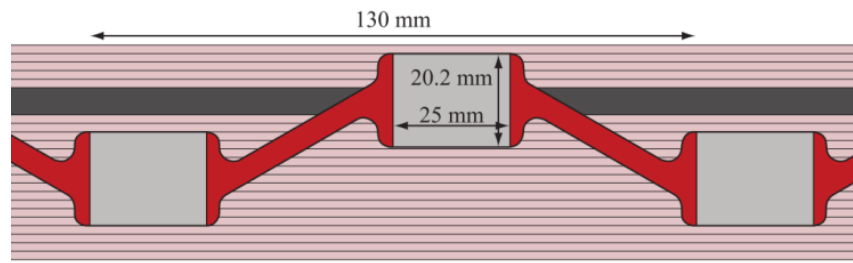

Fig. 3. Design of the MQXF inner layer protection heaters.

\section{Protection heaters delay time}

The protection heaters efficiency is related to how fast they can induce quench within the coils after being fired. The delay time depends on the firing voltage, and on the thickness of the insulation (typically kapton) between the heaters strips and the coils surface, which is $50 \mu \mathrm{m}$ in the case of MQXF. The heaters delay time from firing to induced quench are used in this protection study are showed in Fig. 4. The values are computed using CoHDA [6]. For each layer, two average values are reported: one is related to the high magnetic field (HF) zone (pole block), one is related to the low magnetic field (LF) zone (midplane block).

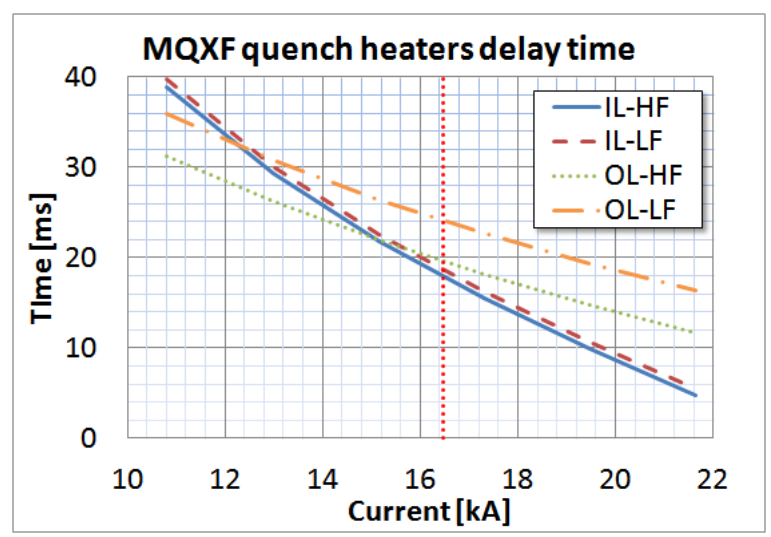

Fig. 4. MQXF quench heaters delay time as a function of the magnet current. The solid curve shows the inner layer high-field delay time, the dashed curve shows the inner layer low-field delay time, the pointed curve shows the outer layer high-field delay time, the dashed-pointed curve shows the outer layer low-field delay time. The operational values are on the pointed vertical line.

\section{HOT SPOT TEMPERATURE}

The hot spot temperature calculation is very important in order to ensure the magnet safety. In fact, during a quench, the temperature of the first resistive zone should not exceed $350 \mathrm{~K}$ [7]. This limit temperature has been established observing experimentally that, below it, there is not permanent degradation in superconducting magnets. Actually, the hot spot temperature could theoretically reach $420 \mathrm{~K}$, beyond which the epoxy impregnation starts to melt; nevertheless, a further $70 \mathrm{~K}$ temperature margin in simulations allows taking into account uncertainties on the protection parameters or material properties.

The hot spot temperature is strongly related to the current decay. Assuming adiabatic conditions the energy per volume unit dissipated by the resistive zone is absorbed by the heat capacity:

$\varrho J^{2} d t=\gamma C_{p} d T$

where $\varrho$ is the resistivity, $J$ the current density, $\gamma$ the cable density and $C_{p}$ the specific heat. From the eq. (1), it is possible to relate the current time integral to a quantity 
which depends only on the conductor material properties, called MIITs:

$$
\operatorname{MIITs}(T)=\int_{0}^{\infty} I^{2} d t=\frac{A^{2}}{10^{6}} \int_{T_{0}}^{T} \frac{\gamma C_{p}(T)}{\varrho(B, T, R R R)} d T
$$

where $A$ is the cross section area, and $T_{0}$ is the magnet operating temperature. This means that, knowing the conductor dimensions and materials, it is possible to calculate the hot spot temperature simulating and integrating the current decay; then, the hot spot temperature is given by the MIITS vs T curve. Note that the MIITs depend on the copper $R R R$ and on the magnetic field where the quench starts, because of the copper magnetoresistivity, which is very effective at low temperatures.

The MQXF conductor parameters can be found in Table 1, and the conductor MIITs calculated at the peak field, considering the cable insulation, are showed in Fig. 5.

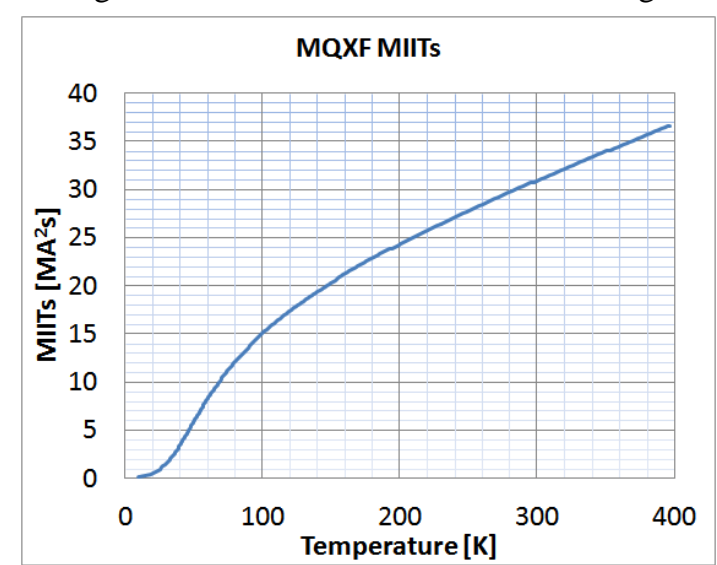

Fig. 5. MIITS vs T curve of the MQXF conductor at peak field (11.4 T).

The material properties used for the calculation are from the MATPRO [8] database. It's easy to see that the limit of $350 \mathrm{~K}$ corresponds to $\sim 34$ MIITs.

The simulations of the current decays have been performed using QLASA [9]. It is a program born for quench simulation in solenoids, but it can be used for accelerator magnets such as quadrupoles with some precautions [10]. The protection parameters used for the simulations are reported in Table II. The main assumptions made are listed below:

- the initial quench is a point (initial size equal to 0 ) located in the peak field zone (pole turn);

- the detection time is computed according to the propagation velocities computed by QLASA, which are based on the Wilson model [11]. It results to be $\sim 7 \mathrm{~ms}$ in order to reach the $100 \mathrm{mV}$ threshold at nominal current;

- the quench heaters delay times are showed in Fig. 4. It is assumed that the quench is induced at a different average time in the high-field and in the low-field blocks of each layer. The quench has null radial dimensions, and it is induced only in the turns actually covered by quench heaters. The heating stations are simulated, but pre-heat from the copper-bridges is not considered (conservative assumption). The induced quench propagates in the radial, azimuthal and longitudinal directions according to the propagation velocities computed by QLASA;

- heat exchange between layers is neglected;

- dynamic effects on the magnet inductance due to the inter-filament coupling currents are taken into account.
These effects have been experimentally observed in the HiLumi experience [1-2]. More details on the electromagnetic model developed and implemented in QLASA can be found in [12];

- quench-back is neglected (conservative assumption).

\section{TABLE II MQXF PROTECTION PARAMETERS}

\begin{tabular}{lc}
\hline \hline Dump resistor (maximum voltage between ends) & $46 \mathrm{~m} \Omega(800 \mathrm{~V})$ \\
Voltage threshold & $100 \mathrm{mV}$ \\
Validation time & $10 \mathrm{~ms}$ \\
Switch opening delay time after validation & $5 \mathrm{~ms}$ \\
\hline \hline
\end{tabular}

The hot spot temperature results are reported in Table III and Table IV. The simulations compare two possible scenarios: in the "1PS" scenario all the triplet magnets are protected in series by one dump resistor and powered by one power supply; in this situation, the total magnetic length is $31.1 \mathrm{~m}$. In the "2PS" scenario Q1 and Q3 are powered and protected separately from Q2a and Q2b;

\begin{tabular}{ccc}
\multicolumn{3}{c}{ TABLE III MQXF HOT SPOT TEMPERATURE / 1PS SCENARIO } \\
\hline \hline Nominal & No IL-QH & Fail OL-QH HF \\
\hline $28.3 \mathrm{MA}^{2} \mathrm{~s}$ & $33.6 \mathrm{MA}^{2} \mathrm{~s}$ & $30.7 \mathrm{MA}^{2} \mathrm{~s}$ \\
$261 \mathrm{~K}$ & $346 \mathrm{~K}$ & $299 \mathrm{~K}$ \\
\hline \hline & TABLE IV MQXF HOT SPOT TEMPERATURE / 2PS SCENARIO \\
\hline \hline Nominal & No IL-QH & Fail OL-QH HF \\
\hline $28.1 \mathrm{MA}^{2} \mathrm{~s}$ & $33.3 \mathrm{MA}^{2} \mathrm{~s}$ & $30.5 \mathrm{MA}^{2} \mathrm{~s}$ \\
$257 \mathrm{~K}$ & $341 \mathrm{~K}$ & $295 \mathrm{~K}$ \\
\hline \hline
\end{tabular}

For each scenario three columns are reported: the first column ("Nominal") shows the hot spot temperature obtained if the protection system (inner and outer quench heaters plus dump resistor) performs as expected; the second column ("No IL-QH") shows the hot spot temperature obtained if there are not Inner Layer Quench Heaters; the last column ("Fail OL-QH HF) shows the hot spot temperature obtained in an unlikely failure scenario, where all the quench heaters of the high-field (pole) blocks do not work, which means that the magnets resistance is $\sim 30 \%$ less than in the nominal case.

Analyzing the results, the first conclusion is that, in the nominal case, the protection of the magnets is ensured; in fact the temperature is well below the limit of $350 \mathrm{~K}$. Then, it is evident that quench heaters on the inner layer are needed in order to ensure protection. A good redundancy is ensured, because removing $\sim 30 \%$ of the magnets resistance gives a temperature of $\sim 300 \mathrm{~K}, 50 \mathrm{~K}$ less than the maximum limit, and this is a very unlikely event. Finally, comparing the results with one or two power supplies, it's easy to note that the two scenarios are very similar. In fact, in both the scenarios the starting decay time constants $\tau=L / R_{d}$ are high ( $\tau=2$ - $4 \mathrm{~s}$ for 2 PS - 1 PS, the total decay time is $\sim 300 \mathrm{~ms}$ ), therefore the current is almost constant when the quench heaters are not yet effective. Then, the decay is dominated by the quench heaters, and the discharge time depends on the resistance per unit length, which is the same in both the scenarios. In conclusion, looking at the hot spot temperature, the option of using just one power supply and one dump resistor for the low- $\beta$ triplet protection circuit should be considered.

Repeating all the simulations without the dynamic effects on the inductance gives $\sim 20 \mathrm{~K}$ more, in all the studied cases. Nevertheless, these effects have been experimentally observed [1-2], the model developed for their simulation using QLASA has been experimentally validated [12], and they are confirmed by an innovative method of magnet inductance measurement during a fast 
current decay [13], therefore they have been included as nominal. Moreover, the reported numbers are probably yet conservative, because it has been showed that quench back, which is not included, strongly affects the fast decays [2].

\section{Peak Voltages}

In this section an analysis of the peak voltages during a quench in MQXF is showed. The study has been performed using ROXIE [14-15], which is a program originally written at CERN for electromagnetic modelling, with a quench simulation subroutine that has been implemented later. ROXIE allows analysing the voltages of each turn during a quench, depending on the current and its derivative, on the resistance, and on the magnetic field. The analysis of peak voltages is very useful in order to dimension and test the insulations of conductors and coils.

The assumptions are basically the same made for the hot spot temperature calculation (see Section III), except that heating station are not simulated, and dynamic effects on the inductance are not taken into account. The simulations compare the scenarios considering one or two power supplies. Nominal case is compared to a good amount of possible quench heaters failure situations. The results are listed in Table V and Table VI. In the 2 PS scenario, numbers are reported only for $\mathrm{Q} 2 \mathrm{a} / \mathrm{Q} 2 \mathrm{~b}$, which is the worst case (longer magnetic length).

The cases considered, from the top to the bottom, are: nominal case; only outer layer quench heaters; failure of all the quench heaters of the first coil (in the coils connection series); failure of all the quench heaters of the third coil; failure of the all the outer layer quench heaters of the highfield (pole) blocks, in all the coils; failure of the inner layer quench heaters of the first coil; failure of the inner layer quench heaters of the third coil; failure of the outer layer quench heaters of the high-field (pole) block of the first coil, just one side (one octant); failure of the outer layer quench heaters of the high-field (pole) block of the third coil, just one side; failure of the outer layer quench heaters of the low-field (mid-plane) block of the first coil, just one side; failure of the outer layer quench heaters of the lowfield (mid-plane) block of the third coil, just one side. For each case, five values are reported: the first is the nominal voltage to ground, computed considering symmetric grounding, i.e. ground positioned in the middle of the magnets chain; the second is the voltage to ground with a short circuit ("sc") of the symmetric ground, i.e. with one of the current leads to ground; the third is the voltage between adjacent turns of the same layer; the fourth is the voltage between adjacent turns of different layers (layer to layer voltage); the fifth is the voltage between the midplane turns. It is evident that the two scenarios are almost equivalent, except to the mid-plane voltage, which doubles in some of the 2 PS scenarios. For this reason, using a suitable mid-plane insulation, the choice of one power supply is convenient also on the peak voltages point of view. The "To Ground" columns need a comment: presently, ROXIE allows simulating only one magnet in a series. This means that the simulations have been performed considering a magnet alone, with a dump resistor dimensioned in order to have the right voltage fall across the magnet ends. Then, the voltage to ground is obtained adding the actual magnet ends input voltage, considering the magnet is inserted in the chain, to the simulated voltage. For this reason, the failure scenarios represent the magnet with a failure in a series of well working magnets, and the case of failures in different magnets cannot be simulated. This causes an error on the to-ground voltage evaluation, of the order of the resistance difference between failure scenario and nominal case.

TABLE V MQXF PEAK VOLTAGES / 1PS SCENARIO

\begin{tabular}{c|ccccc}
\hline \hline & $\begin{array}{c}\text { TG } \\
{[\mathrm{V}]}\end{array}$ & $\begin{array}{c}\text { TG }(\mathrm{sc}) \\
{[\mathrm{V}]}\end{array}$ & $\begin{array}{c}\text { T-T } \\
{[\mathrm{V}]}\end{array}$ & $\begin{array}{c}\text { L-L } \\
{[\mathrm{V}]}\end{array}$ & $\begin{array}{c}\text { M-M } \\
{[\mathrm{V}]}\end{array}$ \\
\hline Nominal & 638 & 838 & 46 & 454 & 148 \\
OL-QH & 757 & 1007 & 86 & 549 & 146 \\
Coil 1 fail & 1862 & 2092 & 62 & 1734 & 1701 \\
Coil 3 fail & 1463 & 1693 & 63 & 1747 & 1832 \\
OL-HF fail & 738 & 958 & 66 & 239 & 148 \\
Coil 1 IL fail & 662 & 872 & 49 & 522 & 356 \\
Coil 3 IL fail & 663 & 873 & 50 & 527 & 482 \\
Coil 1 OL-HF fail 1 side & 608 & 813 & 48 & 487 & 159 \\
Coil 3 OL-HF fail 1 side & 608 & 813 & 47 & 490 & 275 \\
Coil 1 OL-LF fail 1 side & 597 & 802 & 47 & 472 & 147 \\
Coil 3 OL-LF fail 1 side & 582 & 787 & 47 & 472 & 176 \\
\hline \multicolumn{4}{c}{ TABLE VI MQXF PEAK VOLTAGES / 2 PS SCENARIO } \\
\hline Nominal & 659 & 859 & 44 & 421 & 313 \\
OL-QH & 798 & 1048 & 81 & 509 & 311 \\
Coil 1 fail & 1810 & 2020 & 59 & 1674 & 1513 \\
Coil 3 fail & 1335 & 1565 & 59 & 1686 & 1769 \\
OL-HF fail & 833 & 1053 & 62 & 223 & 312 \\
Coil 1 IL fail & 754 & 964 & 47 & 486 & 342 \\
Coil 3 IL fail & 755 & 965 & 47 & 490 & 494 \\
Coil 1 OL-HF fail 1 side & 704 & 909 & 45 & 452 & 314 \\
Coil 3 OL-HF fail 1 side & 704 & 909 & 45 & 455 & 314 \\
Coil 1 OL-LF fail 1 side & 680 & 885 & 45 & 439 & 312 \\
Coil 3 OL-LF fail 1 side & 681 & 886 & 45 & 439 & 313 \\
\hline \hline
\end{tabular}

It is also possible to calculate the maximum coil-toheaters voltage: in the case of grounded heaters, it is equal to the voltage to ground, because the maximum is reached when the heaters are discharged; in the case of floating heaters, the maximum voltage is half of the heaters charge $(500 \mathrm{~V})$ plus half of the voltage between coil ends, because both have a symmetric ground; it is therefore $\sim 650 \mathrm{~V}$. In the case of failure of both the grounds (short-circuits), the maximum coil-to-heaters voltage can reach $\sim 1300 \mathrm{~V}$.

\section{CONCLUSIONS}

The protection of MQXF has been a significant challenge in the first stage of the design process. A first series of studies showed that a standard protection approach (energy extraction and OL heaters) was not sufficient to ensure magnet safety. This result led to improve the design of quench heaters, including them on the inner layer, finding different ways to avoid helium bubbles issue. The current decay behaviour has been studied in deep, leading to develop an electromagnetic model for the simulation of the inductance dependence on the inter-filament coupling currents. The magnet parameters have been upgraded, increasing the margin to the short sample limit. This work shows that, with all these innovations, MQXF protection is possible, with a good margin on the uncertainties (such as material properties), and with a good redundancy because of possible failure scenarios. Moreover, it shows that the option of using only one power supply for the protection of the whole triplet is safe (both hot spot temperature and peak voltages are acceptable), and it could be considered in order to reduce costs. The last open issue is the inner layer quench heaters reliability because of the helium bubbles. If not solved, the option of using CLIQ with outer layer and no inner layer quench heaters should be taken into account. 


\section{REFERENCES}

[1] G. Manfreda, G. Ambrosio, V. Marinozzi, T. Salmi, M. Sorbi and G. Volpini, "Quench Protection Study of the $\mathrm{Nb}_{3} \mathrm{Sn}$ Low- $\beta$ Quadrupole for the LHC Luminosity Upgrade," IEEE Trans. on Appl. Supercond., vol. 24, no. 3, June 2014, Art. No. 4700405.

[2] V. Marinozzi , G. Ambrosio, G. Bellomo, G. Chlachidze, H. Felice, M. Marchevsky, T. Saalmi, M. Sorbi and E. Todesco, "Study of quench protection for the $\mathrm{Nb}_{3} \mathrm{Sn}$ low- $\beta$ quadrupole for the LHC luminosity upgrade(HiLumi-LHC)", IEEE Trans. on Appl. Supercond., vol. 25, no. 3, June 2015, Art. No. 4002905.

[3] E. Ravaioli, V. I. Datskov, G. Kirby, H. H. J. Ten Kate and A. P. Verweij, "A new hybrid protection system for high-field superconducting magnets", Supercond. Sci. Technol., vol. 27, no. 4 044023, 2014

[4] E. Ravaioli et al., "Advanced quench protection for the $\mathrm{Nb}_{3} \mathrm{Sn}$ quadrupoles for the high luminosity LHC," IEEE Trans. on Appl. Supercond., to be published.

[5] G. Ambrosio, et al., "Test Results of the First $3.7 \mathrm{~m}$ Long $\mathrm{Nb}_{3} \mathrm{Sn}$ Quadrupole by LARP and Future Plans," IEEE Trans. Appl. Supercond., vol. 21, no. 3, pp. 1858-1862, June 2011.

[6] T. Salmi, D. Arbelaez, S. Caspi, H. Felice, M. G. T. Mentink, S Prestemon, A. Stenvall, and H. H. J. ten Kate, "A Novel Computer Code for Modeling Quench Protection Heaters in High-Field Nb3Sn Accelerator Magnets", IEEE Trans. on Appl. Supercond., vol. 24, no. 4, 2014, Art. No. 4701810.

[7] E. Todesco, "Quench limits in the next generation of magnets" CERN Yellow Report 2013-006 10-16 (2013).
[8] G. Manfreda, L. Rossi, and M. Sorbi, "MATPRO upgraded version 2012: a computer library of material property at cryogenic temperature," Tech. Rep. INFN-12-04/MI, INFN, April 2012.

[9] L. Rossi and M. Sorbi, "QLASA: A computer code for quench simulation in adiabatic multicoil superconducting windings," Nat. Inst. of Nucl. Phys. (INFN), Rome, Italy, Tech. Rep. TC-04-13,2004.

[10] V. Marinozzi, "Guidelines for the quench analysis of $\mathrm{Nb} 3 \mathrm{Sn}$ accelerator magnets using QLASA," Tech. Rep. TD-13-008, Fermilab Technical Division, 2013.

[11] M.N. Wilson, "Superconducting Magnets", chapter 9, pp. 204-208, Clarendon Press Oxford, 1983.

[12] V. Marinozzi, M. Sorbi, G. Manfreda, F. Bellina, H. Bajas and G. Chlachidze, "Effect of coupling currents on the dynamic inductance during fast transient in superconducting magnets,", Phys. Rev. ST Accel. Beams, vol. 18, no. 3, March 2015.

[13] M. Sorbi, G. Ambrosio, H. Bajas, G. Chlachidze, V. Marinozzi, S. Mariotto and G. Sabbi, "Measurement and analysis of dynamic effects in the LARP model quadrupole HQ02b during rapid discharge," IEEE Trans. on Appl. Supercond., to be published.

[14] N. Schwerg, H. Henke, and S. Russenschuck, Numerical calculation of transient field effects in quenching superconducting magnets. $\mathrm{PhD}$ thesis, Berlin, Berlin, Tech. U., Berlin, 2009. Presented on 18 Nov 2009.

[15] S. Russenschuck, Field Computation for Accelerator Magnets: Analytical and Numerical Methods for Electromagnetic Design and Optimization. Weinheim: Wiley, 2010. 